\section{Zur Wertung aromatischer Nitrokörper als Abortiva.}

Von Dr. J. R. Spinaer in Zürich.

In Nr.46 S. 1272 (1919) dieser Zeitschrift hat H ûbne r einen Fall von Dinitrobenzolvergiftung besprochell, der gewisse Beziehungen zu dem Abortusproblem berührte und in welchem er sich auch aut mich berufell hat. Diese Zitierung erfolgte allerdings offenbar blob auf der Basis eines Referates in der Aerztl. - Sachverständigen-Ztg, die über meine Stellungnahme zu der Abortivwirkung des Nitrobenzols Irrtum erwecken könnte. Seit 10 Jahren habe ich mich mit der Toxikologie gerade des Nitrobenzols eingehend befaßt und 1917 im KorrBlatt für Schweizer Aerzte "Nitrobenzol als Abortivum" besprochen 1) Hübners Stellungnahme zu diesenı Problem nun veranlaBt mich, eipe Klarstellung der Beziehungen zwischen der Verwendung der zu schaffen, um nicht den Glauben an eine Abortivwirkung dieser Körper bei Aerzten und Laien weiterhin aufrechtzuerhalten.

Denn es kann für den Mediziner hier nichts anderes erfolgen als eine reinliche Schendung der Volkspsychologie ${ }^{2}$ ) (als Inbegritf der Einstellung auf Verwendung dieser Körper zu Abortzwecken) von der kritischen Würdigung der toxikologischen Wirksamkeit, welche die notwendige Klarheit schaffen könnte.

H i b n e r druckt sich sehr vorsichtig aus: "Nicht unwahrscheinlich ist auch, daß Dinitrobenzol als Abortivum wirkt," da Menstruationsanomalien, Schmerzen bei der Periode und eitrige Katarrlie be den chronischen Vergiftungen mit dem Mittel beobachtet worden sind. Die Gewährsmänner Hübners sind Fabrikärzte.

Die Arbeiterinnen sollen ihm jedenfalls eine solche abortive Wirkung zuschreiben, er konnte jedoch nicht feststellen, ob das Mittel auch zu derartigen $Z$ wecken genommen worden ist

Damit hat er immerhin die Tatsache wieder erhärtet, daß in Volke ebensosehr, wie auch bei den Medizinern, ein gewisser Glaube, zum mindesten an eine mögliche Wirkung immer noch spukt Dieser Glaube aber ist wiederum der Grund, warum immer und immer wieder Versuche mit diesem Gift unternommen werden. Die Tatsache der öfter mit einem Mittel vorgenommenen Versuche verdichtet sich zum Glauben an die Wirksamkeit ${ }^{3}$ ).

Seit dem unglucklichen Momente, wo die ersten wenig kritischen Ergebnisse einer Magdeburger Abortivepideme mit Nitrobenzol von Schild $d^{4}$ ) publiziert worden sind * und dann in der medizinischen und auch populärmedizinischen Literatur (ich nente hier speziell Fabrice-Weber ${ }^{4}$ ) und Guttzeit $)$ ) die Runde machten, sind ab und $z u$ wieder Fälle sporadisch vorgekommen, die aber, wie ich festzustellen in der Lage war, stets einen gewissen Zusanumeuhang mit dieser Literatur besaßen (vgl. Le ubuscher ${ }^{4}$ ). Der erste vereinzelte Fall stammt aus dem Jalıre 1866, die Schild schen von 1895 .

Es liegt nahe, anzunelımen, daß auf Grund der bekannten Einstellung geu isser industrieller Bevolkerungen auf das Nitrobenzol ene Vebertragung aut der Basis der Namensverwandtschaft bzw. Ab. leitung sich auch auf das Dinitrobenzol erstreckt hat, eine Tatsache, die in der Mittelfindung bei der Abtreibungspsychologie eine große Rolle spielt. Nur auf dieser Basis ist das Uebergreifen aut das Dinitrobenzol als Abortivum zu erklären, und wir haben damit zugleich die Grundlage fur die Bewertung dieser Tatsache.

Ich betrachte es aber nun auf Grund der toxikologischen Forschung als eine dringende Notwendigkeit, den Glauben und die Theorie einer Abortivwirkung der Nitrobenzole zu zerstören. Denn sowohl Mono-

') Spinner, J. R., Schweiz. Jur. Ztg. 1909 H. 9; Pharm. Zentrh. 1913 S. 871 ; Korr. Bl. f. Schweiz. Aerzte 1917 .

2) Die Verhältnisse der Volkspsychologie zu der Auffindung der Abortivmittel werde ich in einer demndchst in dem Arch. f. Sexualforschung erscheinenden Aufsatz: "Psycliologle der
Abtreiber und Abtreibenden ausfuhrlich besprechen.

3) Die fast ebenso rohe Empirie bezüglich der Abortivmittel und Ver. fahren, wie beim niederen Volke, finden wir immer noch bei Aerzten und sogar Sachverständigen, die, weil das Volk ein Mittel anwendet, daraus glauben schließen zu müssen, daß dieses Mittel auch tauglich sei. Ein derartiges Votum befreit sie dann immer leicht von der Notwendigkeit einer genaueren Kausalitătsforschung und macht es sich ebensoleicht, wie dem Juristen bequem. I ch halte dafür, daß wir bezuglich der Wirksamkeit der Volksarbotiva immer noch einen zu wenig kritischen Maßstab an'egen und daB, wie ich in meiner Bearbeitung: Abtreibungshandlungen bei nichtschwangerem Uterus (Vrtljschr. f. gerichtl. M. 58) gezeigt habe, immer noch schwerste Eingriffe be i nicht bestehender Gravidität gemaclit werden. 1) Schild, Sects falle B. kl. W. $1895 \mathrm{~S}$. 187. Fabrice-Weber. Die Lehre von der Kindsabtreibung und rom Kindsmord. Betlin 1911. - Guttzeit, Ein dunkler Punkt.
Lefpzig 1905. - Leubus cher, Vrtjschr. f. gerichti. M. 50 S. 1. als auch Dinitrobenzol sind zu Unrecht in den Ruf von Abortiva gelangt und besitzen nicht die geringsten abortiven Wirkungen.

Die Toxikologie der Nitrobenzole muB in Zusammenhang mit der Gruppe der Methämoglobinbildner gewürdigt werden. Eine ganze Anzahl ab und zu als Abortiva verwendeter Stoffe gehört hierhur. Kaliumchlorat - Pikrinsaure (Trinitiophenol) - Antilebrin (Azetanilid) - Nitro- und Dinitrobenzol - Nitrotoluole - Nitrokresole Nitroglyzerin - Anilin.

Von allen diesen ist jedoch das Mononitrolsenzol oder Mirbanöl (falsches Bittermandelól) das bekannteste Produkt $\left(\mathrm{C}_{6} \mathrm{H}_{5} \mathrm{NO}_{2}\right)$. Vou dem Dinitrobenzol unterscheidet es sich durch den Aggregatzustand, indem es eine ölige Flussigkeit darstellt. Das feste Dinitrobenzol ${ }^{1}$ ) existiert in drei differenten Verbindungen, Ortho-, Meta- und ParaDinitrobenzol, die als isomere Formen aufzufassen sind: $\mathrm{C}_{6} \mathrm{H}_{4}\left(\mathrm{NO}_{3}\right)$ Praktisch kommt jedoch nur die Metaverbindung in Frage, ein gelber gerucliloser, kristallinischer Körper. Wie wir aus der Bearbeitung von $H \ddot{b} b$ ne $r$ ersehen, ist das in diesem Fallc angewendete Präparat ein Gemenge von Kaliumperchlorat mit Innitrobenzol. Daraus ergibt sich eine neue toxikologisch-wichtige Kombination, indem ein bisher nicht als innerliches Gift verwendeter Stoff in diesem Zusammenlang auf einmal zu einer innerlichen Verwendung gelangt. Kaliumperchlorat ist in der toxikologischen Literatı bisher unbekannt, weil nie Fälle von innerlicher Autnalıme vorgekommen sind. Und nun haben wir es hier mit der interessanten Tatsache zu tun, daB eine Kombination zweier Methamoglobinbildner zusammentritt, sodab wir also eine homogen verstärkte Wirkung des Dinitrobenzols annehmen müssen, bei der ein Gehalt an Kaliumperchlorat additionell toxisch wirken mul, weil das Perchlorat $\mathrm{KClO}_{4}$ grumdsätzlich die gleiche Wirkung hat, wie das einfache, bekamte Kaliumchlorat (Kali cliloricum) $\mathrm{KClO}_{3}$, fast der einzige anorganische Methámoglobinbiluner. Rein zutällig sind also hier zwel an sich gleich wirkende Stoffe zusammen eingenommen worden ${ }^{2}$ ).

Nun muß uns generell schon die Tatsache, daß diese Stoffe Blutgitte sind und nur Blutgifte, auf den Gedanken führen, daß sie logischerweise nicht Abortiva scin konnen, dal3 sie keinen spezifischen Einflul auf die Generationsorgane haben konnen. Eine Vergiftuny damit ist immer eine Allgemeinvergiftung, welche das gesamte kreisende Blut betriff. Nach Denning und Kobert, II. 1. c. S.788, tritt der Tod ein, wenn 66 \%o der Blutkörperclien nur noclı Metliämoglobin enthalten. Bis zu dicsem Momente sind aber alle möglichen Zwischenstufen möglich und haben wir die Erscheinungen auf dieser Basis als Stoffwechselstörung und Ernahrulgsstorungen zu bewerten. Daß diese den Uterus und das Nervensystem vorzugsweise affizieren, gibt uns noch immer kein Recht, von eituem tauglichen Abortivun $\measuredangle u$ sprechen. Denn bei diesen Munitionsarbeiterinnen ist auch noch die allgemeine Tatsache zu berücksichtigen, daB im Kriege selır viele Menstruationsstörungen (Kriegsamenorrhoe) vorkanıel und die Spezifizıtat des Dinitrobenzols damit nicht erwiesen ist. Obwohl wir mit Recht annehmen kómuen, daB alle diese Arbeiter an einer subclironischen bis chronischen Methämoglobinvergiftıng leiden, um so mehr, als aucl das Dinitrobenzol zu den Körpern gehört, welche durch die Haut resorbiert werden (Staub) und so ins Blut gelangen. Insofern ist ein Unterschicd zwischen festem Dinitrobenzol und flussigem ollonitrobenzol nicht zu machen.

Num ist aber Dinitrobenzol in der Form eines Sprengstoffes, d. h. zusamnıen mit dem Kaliumperchlorat, genommen worden, wobei $\mathrm{H} \ddot{\mathrm{i}} \mathrm{b}$ ner nun allerdings die Wirkungsmöglichkeit des Kaliumperchlorats bedeutend unterschátzt, weil er annimmt, daB erst cule relativ große Menge im Magen ene bratune Verïarbung be

1) D.s Dinitrofenzol ist, je nachdem es aus gereinigtem (der robem Benz 1 nitriert ist, entweder reines D. oder dann ein Gemisch nuit nitrierten T(luolen und dient fast ausschlieBlich Sprengzwecken (R burit). Akzidentell ist eine Vergiftung damit beobachtet w. rden, wo eine Arbeiterfrau D. mit Insektenpulver gemischt in die Betten gestreut latte (Perkutanaufnahme) Erben II S. 321 häıt das D. eher nocli für giftiger als Mononitrobenzal. Es ist in Wasser nicht loslich, wohl aber in warmen Alkohol, vor allem wird es von der Haut aufgenommen. Es geht zum Teil unzersetat in den Harn über. White (Lancet 1502 S. 59 ) schät7t die t'dliche D. sis für den Menschen auf $\mathrm{ca} .1,8+\mathrm{g}$, ebens Kuric sum mub ich hier einen Verweclislungs-Vergiftungsfall erwähnen, den Weyl in der B.kl. W. 1888 erwähnt hat: Eine Frau nahm zu Abtreibungswecken $3-5 \mathrm{~g}$ des Silffransurr gates Dinitrokres 1 (Viktoriıgelb, orange. Anilinorange) irrtumlicherweise als Safrall und starb innerhali 5 Stunden.

${ }^{3}$ Es darf lier vielleicht auci ein W. rt über den Mißbrauch rin Sprengstoffen zu Genußzwecken gesagt werden. Schon das Glyzerintrinitrin, das als Nitroglyzerin auch in der Therapie $a b$ und $z u$ verwendet wird, fuhrt zu rascher Gewihnung, sodaß die Dosis bis auf $4-7 \mathrm{~g}$ pro Tag gesteigert worden ist. Mit Dynamit cder Sprenggelatine, den teclinischen Verwendungsformen des Nitroylyzerins, sind nun, weil es vin den Arbeitern au: Nasch. haftickeit s,enossen wurde, schon leichtere Vergiftungen vorgekommen. Die gefäßerweiternde Wirkung, die in größeren Dısen haschischartig wird, hat nun $/ \mathbf{u}$ einem spezifischen 1 aster in der elıglisclien Marine gefuhrt, dem Korditessen, wolei der Kordit, ein Gentenge aus 58 Teilen Nitroglyzerin, 37 Teilen Schiebraumwolle und 5 Teilen Vaselin, wie Tabak Igekaut wird. Euphorie und Halluzinatic nen waren die Folge des Genusses, und es war keine leichte Aufgabe fur die Regierung, dieses Laster wieder ein/udämmen. Vgl. v. Sury, Erfahrungen uber Explosionen. Dis;. med. Zurich 1911 S. 51. So konnen wir leicht annehmen, daß vielleicht in Analcgie mit diesen Sprengstoffessen auch andere Sprengstoffe $z$ zu Genul 3 «wecken ausprobiert werden und $z u$ Vergiftungen führen können. 
dingt Beide Körper sind nach den Ergebnissen der Toxikologie in gleichem Maße als Methämoglobinbildner schädlich, wenn sie, wie im vorliegenden Falle, in nerlich eingenommen werden. Ganz anders verhalt es sich bei der perkutanen Resorption, bei welcher fast nur das Dinitrobenzol wirksam wird. Die Mitwirkung des Alkohols als Lösungsmittel allerdings hat dem Dinitrobenzol auclı in diesem Falle bessere Resorptionsmoglichkeiten geschaffen. Dennoch wäre es wohl vollständig irrig, die toxikologische Bedeutung des Kaliumperclilorates in denı Maße zu ignorieren, wie es Hübner getan hat. Aus der Tatsache jedoch, daß wir keinerlei Ergebnisse auf diesem Gebiete in $\operatorname{der}^{\prime}$ Fachliteratur niedergelegt haben, ist diese Stellungnahme erklärlich und eben eine allgemeine Erscheinung, die, wie $\mathrm{Z}$ a n $\mathrm{gg}$ e $\mathrm{r}^{1}$ ) in den Erläuterungen der gewerblichen Gifte oft hervorgehoben hat, dem Arzte erst dann bewuBt und bekannt wird, wenn ein solcher Stoff schon in gröBerem Maße Unheil angerichtet hat. So haben wir rein empirisch auf der Basis der Konstitutionsformel des Alkaliperchlorates anzunehmen, daB es als höhere, labilere Verbindung in erhöhten Maße die Wirkung der Chlorate besitzen muB, daß es ein gefährlicher Methämoglobinbildner ist.

Es ist nun klar, daß in diesem Falle auch das Volk nicht etwa um des Perchlorats willen diesen Sprengstoff als Abortivum betrachtet, sondern daß es darin nur das Dinitrobenzol oder vielmehr bloß das Nitrobenzol sucht. DaB aber das Nitrobenzol ke in taugliclıes, ja nicht einmal relativ taugliches Abortivum ist, glaube ich bereits in meinen früheren Darstellungen hinreichend gezeigt zu haben. In 16 Fälleı trat nur einmal e in wandfrei erwiesen ein Abortus ein, dagegen $7 \mathrm{mal}$ der Tod. Den klinisch bestbeobachteten Fall dieser Reihe hat Roth ${ }^{2}$ ) gegeben, bei welchem die Frau dank der zweckmäßigen Hilfe mit dem Leben davon kam. Roth wies nach, daß die Methämoglobinbildung auch ohne Austritt des Hämoglobins aus den Erythrozyten möglich ist, und beobachtete auch eine starke Gallenfarbstoffreaktion des Blutserums. - Der einzige Fall der Wirkung oder scheinbaren Wirkung ist jedoch nur mit Vorsicht aufzufassen, da wir die möglichen konkurrierenden Ursachen nicht kennen. Immerhin hat dieser Schildsche Fall innerhalb kurzer Zeit in Magdeburg eine Nitrobenzol-Abortivepidemie erzeugt, welcher eine Anzahl von Frauen zum Opfer fielen.

Ebensowenig wie beim Mononitrobenzol kann auch beim Dinitrobenzol von einer spezifischen Abortwirkung gesprochen werden, und man würde als Sachverständiger ein voreingenommenes Urteil abgeben, wenn man dem Volksglauben gemäB das Dinitrobenzol als ein Abortivum wirksamer Art bezeichnen würde.

Denn für die ganze Gruppe der Methamoglobinbildner gilt das grundsätzlich Gleiche: Sie können wohl eine Vergiftung erzeugen, bei der parallel der Mutter auch der Fötus zum Äbsterben kommt, jedoch liegt es in der Natur der Vergiftung als solcher, daB sie auf den Uterus nicht wirken, sondern höchstens der Fötus ebensowohl wie die Mutter an innerer Erstickung zugrundegeht. Dabei scheint es bis jetzt überhaupt noch nicht festgestellt $\mathrm{zu}$ sein, wie weit das Fötalblut methämoglobinisiert wird, oder ob nicht im Plazentarkreislauf eine Verlagerung oder Umsetzung möglich ist, sodaß dann sogar der Fötus eher noch länger lebensfähig bleiben könnte als die Mutter ${ }^{3}$. Diese Verhältnisse bedürfen noch der Forschung auf experimenteller Basis.

Wenn nun die Periodenstörungen in den Dinitrobenzolbetrieben von $\mathrm{Hüb}$ e $r$ hervorgehoben werden, dann haben wir eine Erklärung vielleicht auch darin, da $B$ die Ernährung der Uterusmukosa durch die für den Sauerstoffaustausch untauglich gemachten Erythrozyten nicht mehr in vollkommener Weise erfolgen kann. In gleicher Weise können wir auch teilweise die Wirkung auf das Gehirn erklären, wobei allerdings dann hierbei auch die Wirkung des aromatischen Ringes bei den oìganischen Methämoglobinbildnern nicht außerachtgelassen werden dart.

So komme ich zu dem Ergebnis, daß wir die Methämoglobinbildner in globo so lange nicht als auch nur relativ taugliche Abortiva ansprechen dürfen, bis das Gegenteil auf experimenteller Basis erwiesen ist. ein Nachweis, der uns aber nach den bisherigen Ergebnissen der Erforschung der Methämoglobinbildung durchaus unwahrscheinlich ist. Dem Dinitrobenzol kommt effektiv keine Abortivwirkung zu.

Wenn ich mit den vorstehenden Ausführungen erreicht habe, dab man die Methämoglobinbildner mit mehr Zurückhaltung als Abortiva taxiert und anderseits das sehr labile Kaliumperchlorat nicht mehr als so harmlos betrachtet, dann glaube ich meine Aufgabe erfüllt zu haben. Vielleicht geben diese Zeilen auch Anlaß, daB die Toxikologie des Kaliumperchlorates genauer erforscht wird ${ }^{4}$ ) und bei diesem Anlasse vielleicht auch den Verhältnissen der Methämoglobinbildung in bezug auf den Uebergang in den Fötalkreislauf ein Augenmerk geschenkt wird.

1) Schweiz. Zschr. f. Unfallmed. und Jurisprud. 191 Nr. 9; Rrg. d. Inn. M. u. Kinderhik. V; Zschr, d. naturf. Geselisch. Zürich 1911. - S pinn er, J. R., Arbeiterschutz und gewerbliche

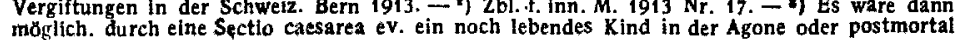
môn erhaiten.

4) Das Kaliumperchlorat, als Kaliumsalz der Ueberchlorsäure, ist erst mit dem Momente ökonomisch wichtig gewcrden, wo es fabrikmalig für Sprengstoffherstellung zu einem akzidentellen Gewerbegift geworden ist, $d$. $h$. einem Gewerbegift, das weniger bel seiner rationellen Verarbeitung als bei Mißbrăuchen Vergiftungen erzeugt. Nach Kobert II S. 770 hat Melckebecke gerade in einer Verunreinigung des Kallumchlorates mit Perchlorat 